\title{
Special Issue on Emerging Approaches to Information Technology and Management, Ingeniería Soldaria, Journal of Engineering and Education, Vol 14, No. 25
}

The twenty-first century is witnessing huge transformation with the help of management and technology innovation. The journal Ingenería Solidaria, subtitled as Journal of Engineering and Education provided us with the opportunity to summon quality research manuscripts on the domain of engineering and management and to compile a special issue. We published the call for papers at IСІткм-2017, the First International Conference on Information Technology And Knowledge Management, organized by Shaheed Sukhdev College of Business Studies, University of Delhi, India, held on December 22-23 2017.

We received more than 35 manuscripts through the open call for papers as well as extended versions of manuscripts presented at ICITKM-2017. After reviewing and screening, eight interesting articles were selected for this special, volume no 14, number 25 of the Journal. We are sure the issue will provide up-to-date discussions and support for readers and researchers in their domains.

The first article was written by Anu Gupta Aggarwal and Aakash, titled "A Multi-attribute Online Advertising Budget Allocation under Uncertain Preferences". It proposes an integrated approach that combines an ordered and weighted averaging operator with fuzzy analytic hierarchy processes for budget allocation. The proposed methodology aids managerial decisions by simultaneously handling multiple attributes through industry experts' opinions, and using a simple proportional rule for allocating budget.

The second article written by Mihir Narayan Mohanty and titled "An Intelligent Approach Based on Embedded System Design for Telemedicine Application" presents an approach in the area of telemedicine for the diagnosis of heart diseases. The model is designed using fuzzy logic in which the rule-based principle is applied to satisfy the objective. The model is developed tacking into account the multi-agent system. The patient's diagnosis is performed using fuzzy inference system.
The next article, written by Pankaj Gupta and Bharat Bhushan Sagar titled "Determining Weighted, Utility-Based Time Variant Association Rules using Frequent Pattern Tree" presents a method to find association rules on time-oriented frequency-weighted, utility-based data, employing a hierarchy for pulling out item sets and establishing their association.

The article by Sameer Anand, Vibha Verma and Anu Gupta Aggarwal titled "2-Dimensional Multi-Release Software Reliability Modeling considering Fault Reduction Factor under Imperfect Debugging" presents a software reliability growth model to assess the reliability of software products released in multiple versions under limited resource and time availability. The model was validated on a real life case of a four-release dataset by carrying out goodness of fit analysis. Laplace trend analysis was also conducted to judge the trend exhibited by data with respect to change in the system's reliability.

The next article was written by Mood Venkanna and Rameshwar Rao, titled "Static Worst-Case Execution Time Optimization using DPSO for ASIP Architecture". It presents a method similar to tree hybridization and path-based methods, which are less precise, and to the global IPET method, which is more precise. Optimization is evaluated with the Discrete Particle Swarm Optimization (DPSO) algorithm for WCET. For several real-world applications involving embedded processors, the proposed technique develops improved instruction sets in comparison to native instruction sets.

The next article, by Arokia Ramya Terrance, Shruti Shrivastava, Asmita Kumari and Lokesh Sivanandam titled "Competitive Analysis of Retail Websites through Search Engine Marketing" presents a comparison and analysis of the apparel category in three competitive retail websites using free search engine optimization tools. This paper focuses on the link between Search Engine Marketing strategies and Search Engine Optimization techniques for 
organic and paid search. It also discusses the impacts of positive and negative website ranking and how such ranking can be improved adopting SEO-friendly practices.

The following article, written by Satya Prakash Yadav and Sachin Yadav titled "Fusion of Medical Images in Wavelet Domain: A Discrete Mathematical Model" makes use of Discrete Wavelet Transform (DWT) for image fusion and extraction of features through images. This approach will help researchers or practitioners in the medical domain to attain better implementations of image fusion and data transmission, which may lead to better treatment procedures and also decrease the data transfer rate as the size diminishes and data loss becomes more manageable.

The last article was written by Prantosh Kumar Paul and Vijender Kumar Solanki. It is titled "Is Virtualization at Present a Cloud Science?" and it highlights several aspects of cloud computing from its beginnings and throughout its growing nature as a field of study with academic and techno-managerial points of view.

We are very thankful with Gloria Jeanette Aponte, Editor at the Universidad Cooperativa de Colombia, in Bogotá, Colombia for the opportunity to edit this special issue for their journal. This preface could not end without a lovingly thank you note for Lorenzo Comini in Italy, for his continuous support in the preparation of this special issue. We are equally thankful with peer reviewers for providing constructive inputs to author's so as to improve their manuscripts. Finally, we must thank our institution's principals CMRIT and SSCBS, for providing a harmonic environment to complete this work in a timely manner.

We hope this special issue based on emerging approaches to Information Technology and Management will provide support to readers and researches for developing their personal work. The greatest care has been taken to prepare an interesting special issue, your feedback is also welcome so as to take into account for future work.

\section{Guest Editors}

Vijender Kumar Solanki, Ph.D. Associate Professor, Department of Computer Science and Engineering, CMr Institute of Technology (Autonomous), Hyderabad, India. Email: spesinfo@yahoo.com

Ajay Jaiswal, Ph.D. Assistant Professor, Department of Information Technology, Shaheed Sukhdev College of Business Studies, University of Delhi, Delhi, India. 


\section{Número especial sobre los enfoques emergentes de Tecnología y gestión de la información, Ingeniería Solidaria, Journal of Engineering and Education, Vol. 14, No. 25}

El siglo XxI está siendo testigo de una gran transformación gracias a la gestión e innovación tecnológica. La revista Ingenería Solidaria, subtitulada Journal of Engineering and Education, nos brindó la oportunidad de convocar manuscritos de investigación de calidad sobre ingeniería y gestión y construir un número especial. Publicamos la convocatoria en ICITKM-2017, Primera Conferencia Internacional sobre Tecnología de la Información y Gestión del Conocimiento, organizada por la Facultad de Ciencias Empresariales Shaheed Sukhdev, de la Universidad de Delhi, India, celebrada del 22 al 23 de diciembre del 2017.

Recibimos más de 35 manuscritos con la convocatoria abierta de trabajos de investigación - también evaluamos versiones extendidas de manuscritos presentados en ICITKM-2017-. Después de revisar y decantar, se seleccionaron ocho artículos interesantes para este número especial, volumen 14, número 25 de la revista. Estamos seguros de que proporcionará discusiones actualizadas y apoyo para lectores e investigadores dedicados a estos dominios.

El primer artículo fue escrito por Anu Gupta Aggarwal y Aakash, titulado "Asignación de presupuesto de publicidad en línea considerando múltiples atributos bajo incertidumbre de preferencias". Propone un enfoque integrado que combina un operador de promedio ordenado y ponderado con procesos de jerarquía analítica difusa para la asignación de presupuesto. La metodología propuesta ayuda a la toma de decisiones gerenciales apoyadas en el manejo simultáneo de múltiples atributos que tienen en cuenta las opiniones de los expertos de la industria y el uso de una regla proporcional simple para asignar el presupuesto.

El segundo artículo, escrito por Mihir Narayan Mohanty y titulado "Aproximación inteligente basada en el diseño de sistemas integrados para la aplicación de la telemedicina" presenta un enfoque centrado en el área de la telemedicina para el diagnóstico de enfermedades cardíacas. El modelo está diseñado mediante una lógica difusa en la que el principio, basado en reglas, se aplica para satisfacer el objetivo. El modelo se desarrolla teniendo en cuenta el sistema multiagente. El diagnóstico del paciente se realiza mediante un sistema de inferencias difuso.

El siguiente artículo, escrito por Pankaj Gupta y Bharat Bhushan Sagar titulado "Determinación de reglas de asociación de variantes de tiempo ponderadas basadas en utilidades mediante la aplicación de un árbol de patrones frecuentes" presenta un método para encontrar reglas de asociación de datos basados en utilidad y ponderados de frecuencia, con el empleo de una jerarquía para extraer conjuntos de elementos y establecer su asociación.

El artículo de Sameer Anand, Vibha Verma y Anu Gupta Aggarwal titulado "Diseño de fiabilidad bidimensional del software de múltiples lanzamientos teniendo en cuenta el factor de reducción de fallas en la depuración imperfecta" presenta un modelo de crecimiento de confiabilidad del software para evaluar la confiabilidad de este tipo, cuyo lanzamiento se hace bajo el modelo de múltiples versiones que presentan limitaciones con respecto a disponibilidad de recursos y tiempo El modelo fue validado mediante un caso real sobre un conjunto de datos con cuatro lanzamientos, mediante un análisis de bondad de ajuste. También se realizó el análisis de tendencia de Laplace para juzgar la tendencia exhibida por los datos con respecto al cambio en la confiabilidad del sistema.

El siguiente artículo fue escrito por Mood Venkanna y Rameshwar Rao, titulado "El peor caso estático de optimización del tiempo de ejecución utilizando DPso para arquitectura AsIP". Este presenta un método similar a la hibridación de árboles y métodos basados en rutas, que son menos precisos, y al método IPET global, que es más preciso. La optimización se evalúa con el algoritmo de optimización de enjambre de partículas discretas (DPSO) para WCET. La técnica propuesta desarrolla conjuntos de instrucciones mejorados en comparación con los conjuntos de instrucciones nativas para varias aplicaciones 
del mundo real que involucran procesadores integrados.

El siguiente artículo, escrito por Arokia Ramya Terrance, Shruti Shrivastava, Asmita Kumari y Lokesh Sivanandam, titulado "Análisis competitivo de sitios web minoristas a través del marketing en motores de búsqueda", presenta una comparación y análisis de la categoría de indumentaria en tres sitios web minoristas competitivos, con el uso de herramientas gratuitas de optimización de los motores de búsqueda. Este artículo se centra en el vínculo existente entre las estrategias de marketing de los motores de búsqueda y las técnicas de optimización para búsqueda orgánica y de pago. Asimismo, analiza los impactos de la clasificación positiva o negativa de sitios web y cómo se puede mejorar dicha clasificación, a partir de la adopción de prácticas de optimización de motores de búsqueda (SEO).

Les sigue un artículo escrito por Satya Prakash Yadav y Sachin Yadav, titulado "Fusión de imágenes médicas en el ámbito de la transformada de ondícula: un modelo matemático discreto", el cual hace uso de la transformada de ondícula (DWT) para la fusión de imágenes y la extracción de características de las mismas. Este ayudará a los investigadores y a los profesionales del campo médico a generar mejores implementaciones de la fusión de imágenes y transmisión de datos, lo que puede conducir a procedimientos de tratamiento más efectivos y también a disminuir la frecuencia de transmisión de datos a medida que el tamaño de los archivos disminuye y la pérdida de datos se vuelve más manejable.

El último artículo fue escrito por Prantosh Kumar Paul y Vijender Kumar Solanki. Se titula "¿Es la virtualización una ciencia de la nube en la actualidad?" y resalta varios aspectos de la computación en la nube, desde sus inicios y en su desarrollo como campo de estudio, con base en puntos de vista académicos y técnico-administrativos.

Estamos muy agradecidos con Gloria Jeanette Aponte, editora de la Universidad Cooperativa de Colombia, en Bogotá, Colombia, por la oportunidad de editar este número especial para su revista. Este prefacio no podría finalizar sin una nota de agradecimiento cariñoso para Lorenzo Comini en Italia, por su continuo apoyo en la preparación de este número. También estamos agradecidos con los revisores pares por proporcionar aportes constructivos a los autores para la mejora de sus manuscritos. Finalmente, debemos agradecer a los directores de nuestra institución CMRIT y SSCBS por proporcionar un ambiente armónico y propicio para que pudiéramos terminar esta asignación de manera oportuna.

Esperamos que este número especial basado en enfoques emergentes sobre tecnología y gestión de la información brinde apoyo y referentes a lectores e investigadores para que desarrollen su trabajo personal. Hemos tenido el mayor cuidado en preparar un número especial que resulte interesante; sus comentarios también son bienvenidos y los tendremos en cuenta para cualquier trabajo futuro.

\section{Editores invitados}

Vijender Kumar Solanki, Ph.D. Profesor asociado, Departamento de Ciencias de la Computación e Ingeniería, Instituto (Autónomo) de Tecnología CMr, Hyderabad, India. Correo electrónico: spesinfo@yahoo.com

Ajay Jaiswal, Ph.D. Profesor asistente, Departamento de Tecnología de la Información, Facultad de Ciencias Empresariales Shaheed Sukhdev, Universidad de Delhi, Delhi, India. 